\title{
Visfatin and Adiponectin Have an Opposite Correlation with Inflammation and Metabolic Syndrome in Non-Diabetic Obese Indonesian Men
}

\author{
Anna Meiliana ${ }^{1,2^{*}}$, Gatot Susilo Lawrence ${ }^{1}$, Ilhamjaya Patellongi ${ }^{1}$, Andi Wijaya ${ }^{1,2^{*}}$. Suryani As'ad ${ }^{1}$
}

\section{Abstract}

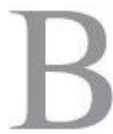

ACKGROUND: Along with the increase in obesity is a parallel increase in the prevalence of metabolic complications of obesity, often referred to as the metabolic syndrome (MetS).The role of adipose tissue in MetS has continued to evolve with the description of numerous secretory peptides from adipocytes named adipocytokines or adipokines. Recent studies have found visfatin as the regulation of inflammatory and immunomodulating processes, meanwhile adiponectin was known to have a potent anti-inflammatory properties. Here we try to assess the correlation between those two adipokines to MetS, via an inflammatory pathway.

METHODS: This was a cross-sectional study on 128 non diabetic obese male subjects (waist circumferences $\geq 90$ $\mathrm{cm}$ ). Visfatin and adiponectin were assessed by ELISA. Statistical analysis was performed using SPSS for Windows v.16.00 with significancy $p<0.05$. The correlations among biomarkers were assessed using Spearman's Rho test.

RESULTS: This study showed a significant positive correlation between levels of visfatin and inflammatory markers TNF- $\alpha(r=0,22, p<0,005)$, and to hsCRP ( $r=$ $0.12, p=0.19)$, significant negative correlation between levels of adiponectin and TNF- $\alpha(r=-0.28, p<0,001)$, adiponectin and $\left.\mathrm{hsCRP}_{\mathrm{S}} \mathrm{r}=-0,14, \mathrm{p}=0.11\right)$ and visfatin $(\mathrm{r}=-.029, \mathrm{p}<0.01)$. Plasma visfatin levels were increased along with the number of MetS components, while plasma adiponectin showed inversely relation.

CONCLUSION: Our present study has shown that visfatin has a proinflammatory properties and adiponectin has an antiinflammatory properties, and how they have an opposite effects on MetS. Visfatin was found to have a positive correlation while adiponectin was found to have a negative correlation with the number of MetS components.

KEYWORDS: Obesity, Inflammation, Metabolic Syndrome, Adipocytokines, Visfatin, Adiponectin, TNF$\alpha$, hsCRP.

\section{Introduction}

Many recent epidemiological studies have documented the rapid increase in the prevalence of obesity. Along with the increase in obesity is a parallel increase in the prevalence of type 2 diabetes, impaired glucose tolerance, and other complications of obesity, such as hypertension, sleep apnea, and arthritis (1).

The metabolic complications of obesity, often referred to as the metabolic syndrome (MetS), according to IDF criteria for men consist of Central Obesity (Waist Circumference $\geq 90 \mathrm{~cm}$ ) plus any two of raised triglyceride (> $150 \mathrm{mg} / \mathrm{dl})$, reduced HDL cholesterol $(<40 \mathrm{mg} / \mathrm{dl})$, raised blood pressure ( $>135 / 85 \mathrm{mmHg}$ ), or fasting plasma glucose $>100 \mathrm{mg} / \mathrm{dl}(2)$. 
The first to suggest a role beyond a repository for lipids for adipose tissue was von Gierke, who in 1905 recognized a role for adipose tissue in glycogen storage. Since the first adipokine, leptin, was discovered in 1994, adipose tissue has been granted many vital roles for the host in general, making it an endocrine organ in its own right (3). The role of adipose tissue in MetS has continued to evolve with the description of numerous secretory products from adipocytes. These "adipokines" are important determinants of insulin resistance, either through a traditional (circulating) hormonal effect, or through local effects on the adipocyte (1).

Visfatin/PBEF/Nampt is a recently discovered 52 $\mathrm{kDa}$ visceral-fat-specific adipokine that is predominantly produced in visceral adipose tissue, particularly by the macrophages. Both its tissue expression and secreted plasma concentrations increase in parallel with obesity (4, 5 ). The first study to identify the potential role of visfatin as an insulin mimetic was by Fukuhara et al (6).

Some other studies have found another function of visfatin is the regulation of inflammatory and immunomodulating processes. Recombinant visfatin activated human leucocytes and induced cytokine production then suggested that adipocyte and macrophagederived visfatin might be an important pro-inflammatory and immunomodulating regulator (7).

Adiponectin secreted exclusively from the adipocytes is a $30 \mathrm{kDa}$ molecule that exists in at least three different higher-order complexes. These complexes have been identified as the high-molecular-weight (HMW) form (1236 mer), low molecular weight (LMW) form (hexamer), and trimeric form (trimer). It is well established that the circulating levels of adiponectin are an important marker for metabolic disorders (8).

AdipoR1 and AdipoR2 serve as major receptors for adiponectin in vivo. They belong to a new family of receptors predicted to contain seven transmembrane domains but to be structurally and functionally distinct from G-protein coupled receptors. AdipoR1 is abundantly expressed in muscle, whereas AdipoR2 is also expressed in liver. Accumulating evidence indicates that AdipoR1 is critical to the anti-inflammatory effects of adiponectin ( 9 , 10).

Several recent reports showed that short term treatment of macrophages with adiponectin first increases the expression of inflammatory cytokines, such as TNF- $\alpha$ and interleukin (IL)-6. In man, the secretion of TNF- $\alpha$ is reported to be mainly due to the cells of the stromal vascular and matrix fractions, including the macrophages, despite the fact that previously most of the mRNA for TNF- $\alpha$ was thought to be found within the adipocytes themselves.
TNF- $\alpha$ is a powerful local regulator within adipose tissue, acting in both an autocrine and a paracrine manner to influence a range of processes. TNF- $\alpha$ playing a pivotal role in relation to the production of several cytokines and other adipokines (11).

An important recent development in our understanding of obesity is the emergence of the concept that it is characterised by a state of chronic low-grade inflammation. The basis for this view is that increased circulating levels of several markers of inflammation, both pro-inflammatory cytokines and acute-phase proteins, are elevated in the obese; these markers include IL-6, the TNF- $\alpha$ system, C-reactive protein (CRP) and haptoglobin (11). In this study, we used TNF- $\alpha$ and hsCRP to present the inflammatory markers.

C-reactive protein (CRP) is a protein found in the blood, the levels of which rise in response to inflammation (an acute-phase protein). CRP is synthesized by the liver in response to factors released by fat cells (adipocytes). It is a member of the pentraxin family of proteins. CRP rises up to 50,000-fold in acute inflammation, such as infection. It rises above normal limits within 6 hours, and peaks at 48 hours. Its half-life is constant, and therefore its level is mainly determined by the rate of production (and hence the severity of the precipitating cause). hsCRP was used to detect a systemic low grade inflammation $(12,13)$.

\section{Methods}

\section{STUDY DESIGN}

This was a cross sectional study using 128 subjects, correlating visfatin and adiponectin and various metabolic profiles to assess the inflammatory properties of each adipokine represented by TNF- $\alpha$ and hsCRP (where concentration of hsCRP $<1$ showed low risk of chronic low-grade inflammation, hsCRP concentration $1-3$ showed moderate risk of chronic low-grade inflammation and hsCRP concentration 3-10 showed high risk of chronic low-grade inflammation) and correlation to the number of MetS components. Data collection was commenced from March - June 2009. The study proposal was approved by the Health Research Ethics Committee of the Faculty of Medicine, University of Hasanuddin, Makassar.

\section{SUBJECTS}

The study subjects comprised employees of PT Showa Indonesia and PT Posmi Indonesia, Cikarang who met the inclusion criteria, they were apparently healthy, male, 
aged $30-60$ years, with waist circumferences $\geq 90 \mathrm{~cm}$, willing to participate in this study, and agreed to follow the study protocols. Each subject was given explanation about the study, and signed informed consent prior to the commencement of the study.

Subjects who were consuming anti-inflammatory drugs such as statins or thiazolidinedione during the last 3 weeks; had diabetes with fasting plasma glucose $>126 \mathrm{mg} /$ dl, had liver dysfunction, kidney dysfunction, asthma, and acute inflammation (hsCRP $>10 \mathrm{mg} / \mathrm{dl}$ ) were excluded from the study.

\section{ANTHROPOMETRIC MEASUREMENT}

Body weight (BW) was measured in kilograms to the nearest $0.1 \mathrm{~kg}$, with light clothes on, using a beam scale Tanita (Tanita, Japan). Height (Ht) was measured in centimeters to the nearest $0.1 \mathrm{~cm}$, in standing position with socks and shoes removed, using a microtois (stature meter). Waist circumference was measured in centimeters to the nearest $0.1 \mathrm{~cm}$, using a flexible non-elastic tape made by Roche (Roche, Switzerland). The Waist Circumferences was measured at the part of the trunk located midway between the lower costal margin (bottom of lower rib) and the iliac crest (top of pelvic bone) while the person was standing, with feet about $25-30 \mathrm{~cm}$ apart. The measurer should stand beside the individual and fit the tape snugly, without compressing any underlying soft tissues. The circumference should be measured to the nearest $0.5 \mathrm{~cm}$, at the end of a normal expiration.

\section{BIOCHEMICAL ASSESSMENT}

Subjects were told to be fasting for 12 hours at the night before sample collection. Fasting blood samples were collected in the morning between 07:00 to 10:00 am. Plasma and serum were separated immediately by centrifugation and aliquots were frozen at $-200 \mathrm{C}$ for subsequent batched analysis for visfatin, adiponectin, and hsCRP.

Serum triglycerides, total cholesterol (TC), low and high-density lipoprotein cholesterol (LDL-C and HDL -C), Fasting Plasma Glucose, creatinine, alanine amino transferase (ALT) and aspartate amino transferase (AST) levels were assayed by the Prodia Clinical Laboratory's routine chemistry procedures. Serum AST, ALT and creatinine were determined using the International Federation of Clinical Chemistry (IFCC) method and reagents manufactured by Roche (Mannheim, Germany). The Standard automated glucose oxidase method was used to determined glucose, reagents manufactured by Roche (Mannheim, Germany). Homogeneous method was used to determine HDL-C, using reagents manufactured by Daiichiß Tokyo-Japan. The phosphate glycerol oxidase- peroxidase anti-peroxidase (GPO-PAP) method was used to determine triglycerides, using reagent manufactured by Roche (Mannheim, Germany).

Plasma visfatin was determined using an enzymelinked immunoassay (ELISA) kit from Adipogen, Inc. Seoul, Korea, and plasma adiponectin was determined using ELISA kit from Daiichi, Japan. TNF- $\alpha$ was determined using ELISA ELISA kit from R\&D Systems, Inc. Minneapolis, USA.

A high-sensitivity CRP (hs-CRP) test measures low levels of CRP using laser nephelometry. The test gives results in 30 minutes with a sensitivity down to $0.04 \mathrm{mg} / \mathrm{L}$. Normal concentration in healthy human serum is usually lower than $10 \mathrm{mg} / \mathrm{L}$ Other biochemical markers were assessed soon after plasma separation.

\section{DATA ANALYSIS}

Data analysis was done using SPSS 16.0 statistical analysis software for Windows (SPSS Inc., Chicago, IL, USA). Distributions of continuous variables were assessed for normality using the Kolmogorov-Smirnov. For continuous variables with normal distributions, such as anthropometric and biochemical measures, descriptive statistics were presented as mean $\pm \mathrm{SD}$. Associations between continuous variables with approximately normal distributions were described using Spearman's correlation coefficients. All tests were two-sided and considered significant at $\mathrm{p}<$ 0.05 .

\section{Results}

Table 1 shows the general description of subjects' baseline characteristics. Subjects aged averagely 38.23 years, with waist circumferences means $97.6 \pm 5.72 \mathrm{~cm}$. Subjects included in this research had no hypertension (blood pressure systolic means $114.36 \pm 5.28$ and diastolic $75.78 \pm$ 10.16), no diabetes (Fasting Plasma Glucose means 89.90 \pm 8.65 ), no liver enzyme impaired (AST means $29.86 \pm$ 9.17 , ALT $39.3 \pm 16.64$ ), no renal dysfunction (creatinin means $1.09 \pm 0.15$ ), and no acute inflammation (hsCRP means $2.34 \pm 1.85$ ).

Table 2 shows a significant correlation between visfatin and TNF- $\alpha$, positive but not significant correlation between visfatin and hsCRP, and also with number of MetS components. The trend was also shown in Figure 1(A), where higher concentration of plasma visfatin was found along with the number of MetS components. In the other way, Plasma adiponectin was strongly negative 
correlated with TNF- $\alpha$, and negatively correlated even though not significant with hsCRP and the number of MetS components. In figure 1(B) it was shown the trend of decreased plasma adiponectin concentration along with the number of MetS components. The correlation between visfatin and adiponectin was found to be significantly negative $(\mathrm{p}<0.01)$.

Table 1. Description of Subjects Baselines Charateristics.

\begin{tabular}{|c|c|c|c|c|c|c|}
\hline \multirow[t]{2}{*}{ Variables } & & Min & Max & \multicolumn{3}{|c|}{ Means } \\
\hline & & \multicolumn{2}{|c|}{$(n=128)$} & & & \\
\hline Age & (years) & 30 & 53 & 38.23 & \pm & 5.50 \\
\hline \multicolumn{7}{|l|}{ Clinical Variables } \\
\hline WC & $(\mathrm{cm})$ & 91 & 126 & 97.6 & \pm & 5.72 \\
\hline Height & $(\mathrm{cm})$ & 156 & 185 & 166.36 & \pm & 5.28 \\
\hline Weight & $(\mathrm{Kg})$ & 66 & 120 & 80.31 & \pm & 8.02 \\
\hline SBP & $(\mathrm{mmHg})$ & 90 & 150 & 114.36 & \pm & 13.19 \\
\hline DBP & $(\mathrm{mmHg})$ & 60 & 110 & 75.78 & \pm & 10.16 \\
\hline \multicolumn{7}{|c|}{ Biochemical Variables } \\
\hline Bilirubin & $(\mathrm{mg} / \mathrm{dl})$ & 0.07 & 0.42 & 0.23 & \pm & 0.06 \\
\hline AST & $(\mathrm{U} / \mathrm{l})$ & 17 & 60 & 29.86 & \pm & 9.17 \\
\hline ALT & $(\mathrm{U} / \mathrm{l})$ & 11 & 90 & 39.3 & \pm & 16.64 \\
\hline Creatinin & $(\mathrm{mg} / \mathrm{dl})$ & 0.6 & 1.4 & 1.09 & \pm & 0.15 \\
\hline FPG & $(\mathrm{mg} / \mathrm{dl})$ & 70 & 119 & 89.80 & \pm & 8.65 \\
\hline LDL & $(\mathrm{mg} / \mathrm{dl})$ & 39 & 313 & 139.24 & \pm & 37.49 \\
\hline Triglyceride & $(\mathrm{mg} / \mathrm{dl})$ & 42 & 1312 & 192.77 & \pm & 134.86 \\
\hline $\mathrm{HDL}$ & $(\mathrm{mg} / \mathrm{dl})$ & 29 & 71 & 44.38 & \pm & 7.81 \\
\hline hsCRP & $(\mathrm{mg} / \mathrm{l})$ & 0.24 & 9.12 & 2.34 & \pm & 1.85 \\
\hline Visfatin & (ng/ml) & 0.06 & 5.63 & 1.58 & \pm & 1.16 \\
\hline Adiponectin & (ng/ml) & 831.69 & 5495.79 & 3026.39 & \pm & 961.16 \\
\hline TNF- $a$ & (ng/ml) & 1.33 & 46.48 & 3.38 & \pm & 4.40 \\
\hline
\end{tabular}

Description: $\quad W C=$ Waist Circumference

SBP = Systolic Blood Pressure

DBP $=$ Diastolic Blood Pressure

AST $=$ Aspartate Aminotransferase

ALT = Alanin Aminotransferase

FPG = Fasting Plasma Glucose

HDL-C = High Density Lipoprotein Cholesterol

LDL-C = Low Density Lipoprotein Cholesterol

HOMA IR = Homeostasis Model Assessment of Insulin Resistance

TNF- $\alpha=$ Tumor Necrosis Factor $-\alpha$

hsCRP = high sensitivity C-reactive protein . 
Table 2. Correlation between variables.

\begin{tabular}{|l|c|c|}
\hline & $\mathbf{r}$ & $\mathbf{p}$ \\
\hline Vistafin vs & & \\
TNF- $\alpha$ & $0.22^{*}$ & 0.02 \\
hsCRP & 0.12 & 0.19 \\
Number of MetS components & 0.09 & 0.33 \\
Adiponectin & $-0.28^{* *}$ & 0.00 \\
\hline Adiponectin vs & & \\
TNF- $\alpha$ & $-0.26^{*}$ & 0.00 \\
hsCRP & -0.14 & 0.11 \\
Number of MetS components & -0.14 & 0.12 \\
\hline
\end{tabular}

Description: ${ }^{\star}=$ significant at $p<0.05 ;{ }^{\star \star}=$ significant at $p<0.01$.

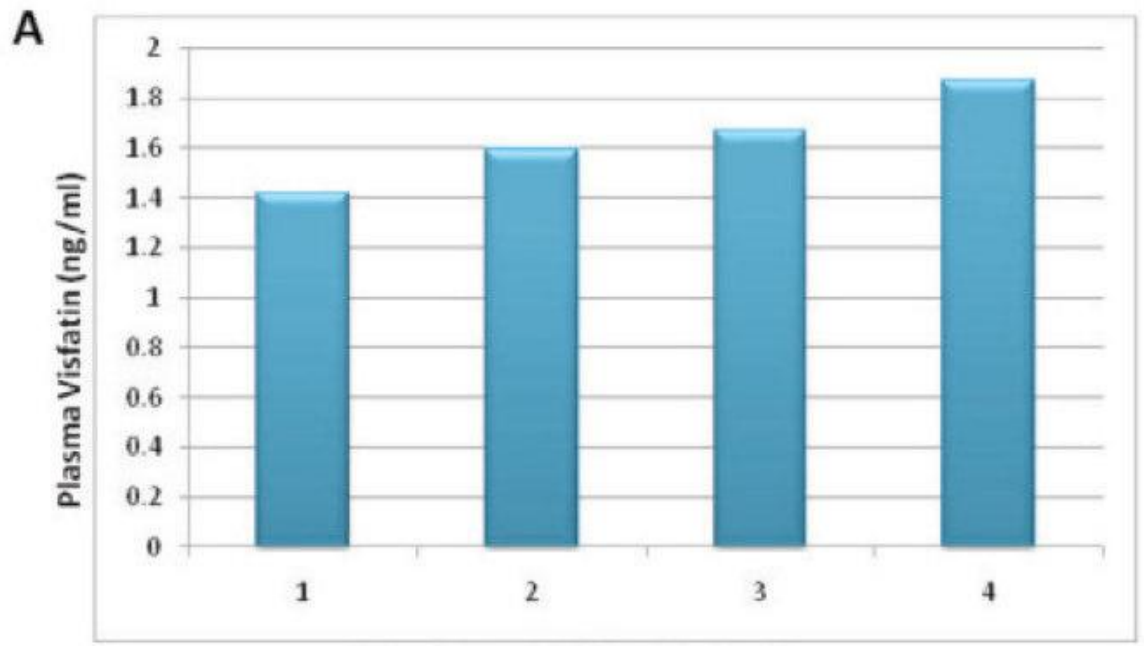

Number of MetS Components

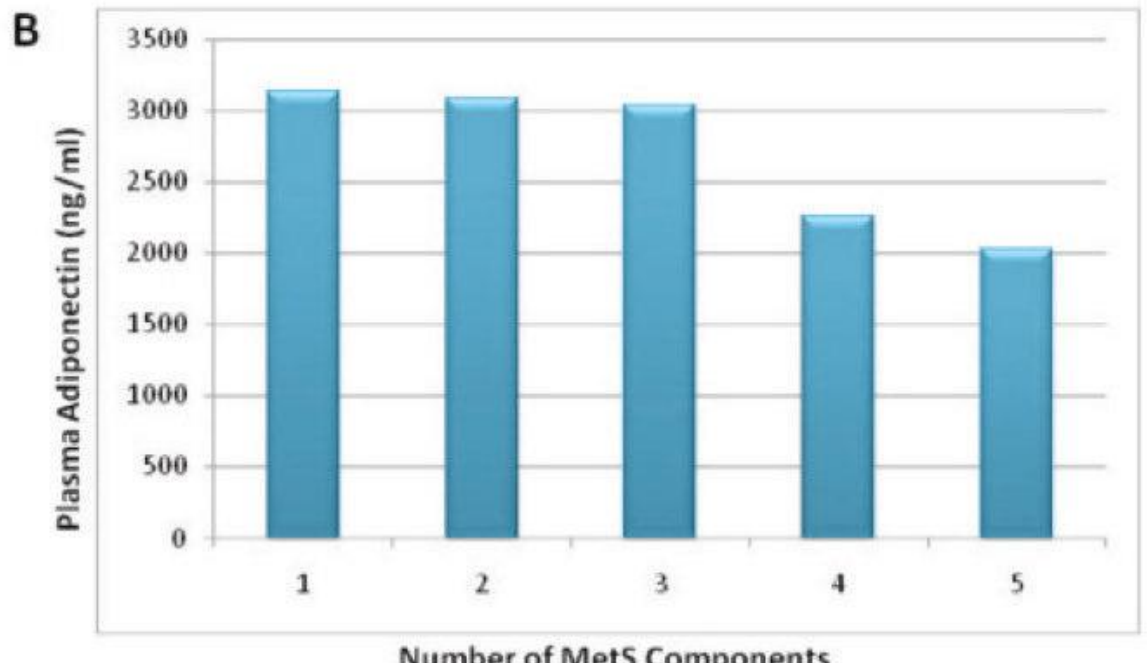

Number of Mets Components

Figure 1. Plasma Visfatin and Adiponectin according to the number of MetS components. 


\section{Discussion}

This study was carried out to assess the role of visfatin and adiponectin in the number of MetS components, via an inflammatory pathway. Subjects were limited only to men to prevent any gender bias, as we know that the adiposity was higher in women then most adipocytokines was also expressed higher including visfatin. (14).

In this study, waist circumferences were found to be positively correlated with visfatin and negatively correlated with adiponectin. Visfatin was predominantly found in visceral adipose tissue, from which the name visfatin was derived. In human studies, a positive correlation between visceral adipose tissue visfatin gene expression was noted (1).

Increased WC describes the enlargement of visceral adipose tissues, Visceral obesity is associated with macrophage infiltration, a finding that has shed a new light in the understanding of obesity-related inflammation, initiating a state of cellular stress and activation of proinflammatory pathways, especially nuclear factor- $x \mathrm{~B}$ (NF$x \mathrm{~B})$. This results in upregulated adipocyte production of pro-inflammatory adipokines, including visfatin (9).

Adiponectin and TNF- $\alpha$ exert a negative reciprocal intractions on their local production in AT. In obese subjects, low grade inflammation occurred in adipose tissue increased the expression of TNF- $\alpha$, which via a NF- $x \beta$ pathway could inhibit adiponectin secretion. This decreased production of adiponectin in obesity may aggravate inflammation in AT, thereby creating a "vicious circle" to lower adiponectin release (9). This negative correlation also showed in table $3(\mathrm{r}=-0.26 ; \mathrm{p}<0.01)$.

The positive correlation between visfatin with TNF- $\alpha$, and also visfatin with hsCRP showed the proinflammatory properties of visfatin. A study by Moschen AR has shown that recombinant visfatin activates human leucocytes and induces cytokines production. They found that visfatin induced $\mathrm{p} 38$ activation in pre B-cell formation. Visfatin also upregulated NF- $x \beta$ p 65 DNA - binding activity in human leucocyte (7). Similar to the study by Fillipatos et al, in this study we found increased plasma visfatin along with the number of MetS components, supporting the suggestion that visfatin could be considered as a proinflammatory cytokine (15).

In the study of Zhang P et al, they found that TNF- $\alpha$ significantly decreased the mRNA expression of AdipoR1 in endothelial cells, which suggests a potential role in endothelial dysfunction and adiponectin resistance so that hypoadiponectinemia commonly seen in obesity and type 2 diabetes. NF- $x \beta$ is an important transcription factor that mediates the induced expression of proinflammatory adhesion molecules in response to TNF- $\alpha$. TNF- $\alpha$ stimulates the protein degradation of $I x \beta$, the inhibitor of NF- $x \beta$, which leads to NF- $x \beta$ nuclear translocation and subsequent transcriptional activation of the target genes. Adiponectin inhibits the TNF- $\alpha$ activation by preventing the I $x \beta$ degradation and NF- $x \beta$ activation, which may contribute to the receptors' antiinflammatory effect (16, 17)

In this study, the plasma adiponectin was found to be decreased along with the number of MetS components. On the same inflammation basis, a study by Xydakis AM et al showed that plasma adiponectin level was significantly lower in MetS subjects as compared with age- and BMImatched non MetS- individuals (18).

\section{Conclusions}

Ourpresent study showed that visfatinhad a proinflammatory properties and adiponectin had an antiinflammatory properties, and how they had an opposite effects on MetS. Visfatin was found to have a positive correlation while adiponectin was found to have a negative correlation with the number of MetS components.

\section{Acknowledgements:}

This study was fully funded by the Prodia Foundation for Research and Training. We are indebted to the technical staff of the Prodia Clinical Laboratory, Clinical Trial, Research and Development Departments for their valuable assistance in undertaking specimen collection in the field and biochemical assessments.

\section{References:}

1. Rasouli N, Kern PA. Adipocytokines and the Metabolic Complications of Obesity. J Clin Endocrinol Metab 2008; 93: S64-S73.

2. Alberti KGM, Zimmet $P$, Shaw J, for he IDF Epidemiology Task Force Consensus Group. The metabolic syndrome-a new worldwide definition. Lancet 2005; 366: 1059 1062.

3. Wozniak SE, Gee LL, Wachtel MS, Frezza EE. Adipose tissue: the new endocrine organ? A review article. Dig Dis Sci. 2009; 54: 1847 - 1856.

4. Lee WJ, Wu CS, Lin $\mathrm{H}$, et al. Visfatin-induced expression of inflammatory mediators in human endothelial cells through the NF-kß pathway. Int J Obes 2009; 33: 465-472. 
5. Korner A, Garten A, Bluher M, et al. Molecular Characteristic of Serum Visfatin and Differential Detection by Immunoassay. J Clin Endocrinol Metab 2007;

6. Fukuhara A, Matsuda M, Nishizawa M, et al. Visfatin: A Protein Secreted by Visceral Fat that Mimetics the Effects of Insulin. Science 2005; 307: 426.

7. Moschen AR, KaserA, Enrich B, et al. Visfatin, an Adipocytokine with Proinflammatory and Immunology Properties. J Immunol 2007; 178: 1748 - 1758.

8. Schraw T, Wang ZV, Halberg N, Hawkins M, Scherer PE. Plasma Adiponectin Complexes Have Distinct Biochemical Characteristics. Endocrinology 2008; 149: 2270-2282.

9. Maury E, Brichard SM. Adipokine dysregulation, adipose tissue inflammation and metabolic syndrome. Mol Cell Endocrinol 2010; 314: 1 - 16 .

10. Park PH, Huang $H$, McMullen MR, Manda $P$, Sun L, Nagy LE Suppression of Lipopolysaccharide-stimulated Tumor Necrosis Factor- $\alpha$ Production by Adiponectin Is Mediated by Transcriptional and Post-transcriptional Mechanisms. J Biol Chem 2008; 283: 26850-26858.

11. Trayhurn P, Wood IS. Adipokines: inflammation and the pleiotropic role of white adipose tissue. $\mathrm{Br} \mathrm{J}$ Nutr 2004; 92: $347-355$.
12. Pepys MB, Hirschfield GM. C-reactive protein: a critical update. J Clin Invest 2003; 111: 1805 - 1812.

13. Clyne B, Olshaker JS. The C-reactive protein. J Emerg Med 1999; 17: 1019-1025.

14. Ibánéz L, Sebastiani G, Lopez - Bermejo A, Diaz M, Gomez - Roig MD, de Zegher F. Gender Specificity of Body Adiposity and Circulating Adiponectin, Visfatin, Insulin, and Insulin Growth Factor-I at Term Birth: Relation to Prenatal Growth. J Clin Endocrinol Metab 2008; 93: 2774-2778.

15. Filippatos TD, Derdemezis CS, Gazi IF, et al. Increased plasma visfatin levels in subjects with the metabolic syndrome. Eur J Clin Invest 2008; 38: 71-72.

16. Zhang $P$, Wang $Y$, Fan $Y$, Tang $Z$, Wang $N$. Overexpression of Adiponectin Receptors Potentiates the Antiinflammatory Action of Subeffective Dose of Globular Adiponectin in Vascular Endothelial Cells. Arterioscler Vasc Thromb Biol. 2009; 29: 67-74.

17. Ouchi N, Walsh K. A Novel Role for Adiponectin in the Regulation of Inflammation. Arterioscler Vasc Thromb Biol. 2008; 28: 1219 - 1921.

18. Xydakis $\mathrm{AM}$, Case $\mathrm{CC}$, Jones $\mathrm{PH}$, et al. Adiponectin, Inflammation, and the Expression of the Metabolic Syndrome in Obese Individuals: The Impact of Rapid Weight Loss through Caloric Restriction. J Clin Endocrinol Metab 89: 2697 - 2703. 